\title{
Defecto del canal auriculoventricular, aurícula única y atresia tricuspídea como parte de un caso de síndrome de Ellis-Van Creveld
}

\author{
Atrioventricular canal defect, single atrium and tricuspid atresia as part of a \\ case of Ellis-Van Creveld syndrome
}

\author{
Dra. Sara Nila Gonzales Portillo ${ }^{a}$ Dra. Rosa Conde Sumire ${ }^{a}$, Dr. Fernando Gamio Vega Centeno ${ }^{b, c}$, \\ est. Gustavo Hernández-Córdovac y Dr. Franco Romaní Romaníd,e
}

\begin{abstract}
RESUMEN
El síndrome de Ellis-Van Creveld (SEVC) o displasia condroectodérmica se debe a una mutación de transmisión autosómica recesiva en el brazo corto del cromosoma 4 y afecta múltiples órganos. Descrito como una tétrada clásica de condrodisplasia, displasia ectodérmica, polidactilia y defectos cardíacos congénitos, sólo se conoce a partir de informes y series de casos. Se describe el caso de un varón de 3 meses, sin antecedentes familiares de importancia, que presentó un cuadro de condrodisplasia, labio superior fusionado al paladar, simpolidactilia posaxial bilateral en las manos, displasia del desarrollo de las caderas, tórax estrecho con costillas cortas y compromiso cardíaco. El presente caso sería la primera comunicación del SEVC en el Perú. Palabras clave: síndrome de Ellis-Van Creveld, condrodisplasia, aurícula única, polidactilia posaxial.
\end{abstract}

\begin{abstract}
Ellis-Van Creveld Syndrome or chondrectodermal dysplasia is produced by an autosomal recessive inheritance secondary to mutation in the short arm of chromosome 4 . The syndrome affects multiple organs. It is described as a clinical tetrad that involves chondrodysplasia, ectodermal dysplasia, polydactyly and congenital heart defects. It is only known from reports and case series. We present a three months old male, without relevant family history, who presented chondrodysplasia, upper lip merged to palate, bilateral sinpolydactyly in the hands, developmental dysplasia of the hip, narrow chest with short ribs, and heart defects. This case is the first report of EVC in Peruvian literature.

Key words: Ellis-Van Creveld Syndrome, chondrodysplasia, single atrium, postaxial polydactyly.
\end{abstract}

http:/ /dx.doi.org/10.5546/aap.2013.e58

a. Hospital Regional del Cusco, Servicio de Pediatría B. Cusco, Perú.

b. Hospital Adolfo Guevara Velasco EsSalud-Cusco. Servicio de Cardiología.

c. Universidad Nacional de San Antonio Abad del Cusco, Cusco, Perú.

d. Oficina General de Investigación y Transferencia Tecnológica, Instituto Nacional de Salud, Lima-Perú.

e. Departamento de Medicina Preventiva y Salud Pública, Facultad de Medicina, Universidad Nacional Mayor de San Marcos.

Correspondencia:

Gustavo Hernández Córdova: hernndezjorgegustavo@gmail.com

Conflicto de intereses: Ninguno que declarar.

Recibido: 28-9-2012

Aceptado: 20-11-2012

\section{INTRODUCCIÓN}

El síndrome de Ellis-Van Creveld (SEVC), denominado también displasia condroectodérmica o displasia mesoectodérmica (OMIM 225500), es una displasia esquelética autosómica recesiva. ${ }^{1,2}$ El fenotipo es variable y afecta múltiples órganos. Sus características clínicas se describieron en numerosos informes ${ }^{3-6}$ y series de casos. ${ }^{7-12}$ Después del nacimiento, el cuadro clínico suele ser la tétrada conformada por: 1) estatura pequeña con acortamiento de la parte distal de los miembros y acortamiento de las falanges media y distal; 2) polidactilia en las manos (casi siempre bilateral), que ocasionalmente afecta los pies; 3) displasia condroectodérmica que afecta principalmente las uñas, el cabello y los dientes, y 4) malformaciones cardíacas congénitas ( $50 \%$ a $60 \%$ de los casos); las más frecuentes son defectos septales y aurícula única; también se han informado defectos de las válvulas mitral y tricúspide, persistencia del conducto arterioso y síndrome del corazón izquierdo hipoplásico. ${ }^{1,2,7}$

La presencia de malformaciones cardíacas apoya el diagnóstico de SEVC y parece ser el principal determinante de la longevidad de los pacientes afectados; se las asocia con una altísima mortalidad. Uno de cada dos pacientes con SEVC y afección cardíaca fallece en la infancia; los sobrevivientes pueden tener una expectativa de vida normal. ${ }^{13}$ El diagnóstico definitivo es molecular y se basa en la homocigosidad para una mutación en los genes EVC1 o EVC 2 a nivel de 4p16 mediante secuenciación directa. ${ }^{1}$

La prevalencia exacta se desconoce; sin embargo, al nacimiento se ha estimado en 7 casos por 1000000 de personas. ${ }^{6,7}$ Una revisión de 2007 informó que desde su descripción inicial, en el año 1940, se habían publicado 150 casos en la bibliografía. ${ }^{1}$ Desde 2007, se han publicado unos 60 informes o series de casos (disponible en www.pubmed.com. Consultado el 20 septiembre de 2012). 
Se presenta el caso de una enfermedad infrecuente, comunicada por primera vez en Perú; su valor educativo muestra el cuadro clínico clásico del síndrome de Ellis-Van Creveld.

\section{INFORME DE CASO}

Un varón de 3 meses, con malformaciones congénitas múltiples, fue hospitalizado en el Hospital Regional del Cusco con diagnóstico de neumonía extrahospitalaria. Los padres eran agricultores, sanos, no había consanguinidad ni referían antecedentes de enfermedades congénitas en otros familiares. Como antecedentes patológicos de los padres, un año antes habían recibido un curso de tratamiento con estibogluconato de sodio contra la leishmaniosis cutánea.

En el momento de la concepción la madre tenía 16 años y durante la gestación se realizó seis controles. El parto se produjo en el Hospital Regional de Cusco, en presentación podálica por vía vaginal, por ingresar la madre en fase expulsiva. El producto fue un recién nacido a término de 2600 gramos, con un puntaje de Apgar de 6 y 8 al primero y quinto minuto.
En el examen físico se constató enanismo, labio superior fusionado al paladar, dientes y uñas displásicos, tórax estrecho con costillas cortas, simpolidactilia posaxial bilateral con siete dedos en la mano derecha, hexadactilia en la mano izquierda y genu varo en los miembros inferiores (Figura 1).

La radiografía anteroposterior de tórax mostró cardiomegalia. La ecocardiografía transtorácica evidenció un defecto del canal auriculoventricular, aurícula única y atresia tricuspídea, así como una comunicación interventricular que compensaba hemodinámicamente el cuadro. En la ecografía de caderas se encontró un déficit en el desarrollo de tipo IIA. No se realizó el estudio citogenético. No se hallaron otras alteraciones de laboratorio significativas.

Durante la evolución, el paciente se mantuvo hemodinámicamente estable y no presentó hipertensión pulmonar. Se recomendó a los padres evitar situaciones que incrementaran el gasto cardíaco, incluidos los viajes a ciudades de gran altitud. El paciente fue referido para evaluación especializada a un establecimiento de mayor capacidad resolutiva.

Figura 1. A y B. Polidactilia bilateral de las manos. C. Labio superior fusionado al paladar. D. Fotografía frontal del paciente.

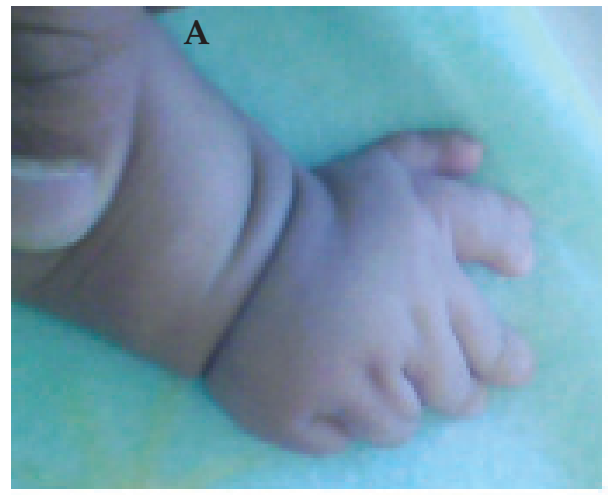

B
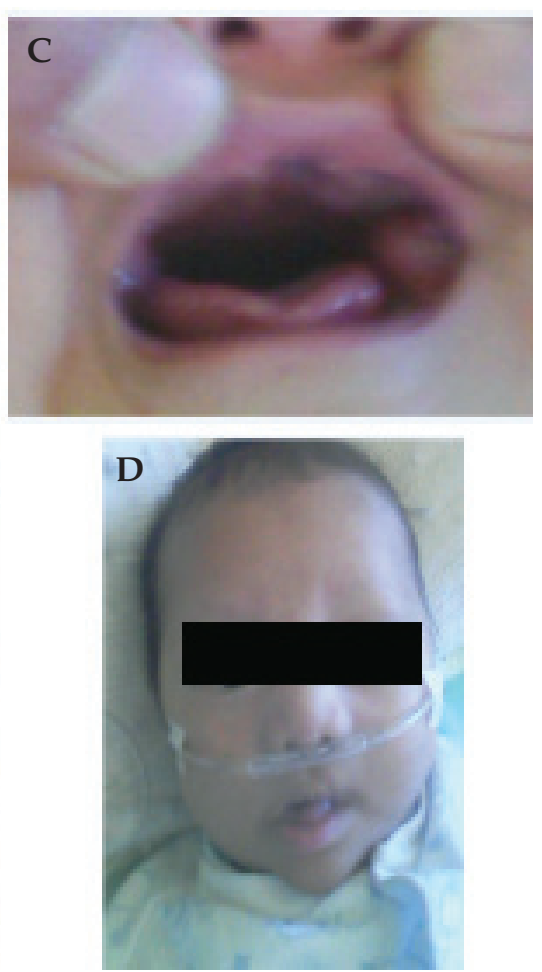


\section{DISCUSIÓN}

El síndrome de Ellis-Van Creveld es una rara enfermedad autosómica recesiva que afecta por igual a hombres y mujeres..$^{1-3}$ Se presenta con mayor frecuencia en familias con historia de consanguinidad. En el caso analizado no había ese antecedente. El paciente era el primer hijo de una madre adolescente y contó con seis controles prenatales. No se pudo determinar la realización de una ecografía en dichos controles. El diagnóstico prenatal puede realizarse mediante ecografía: la presencia de defectos en la estructura fetal al final del primer trimestre, incluidos tórax estrecho, acortamiento de huesos largos, metáfisis redondeada, polidactilia posaxial en manos o pies, y defectos cardíacos sugiere su diagnóstico. ${ }^{1}$

Venkat-Raman y cols. encontraron un incremento del grosor de la translucidez nucal fetal en el primer trimestre, que ha sido asociada con el SECV durante la semana 13 de gestación.

Parte del consejo genético que se le debe brindar a la madre, en el caso de este paciente, es que el riesgo para futuros embarazos es del $25 \%{ }^{1} \mathrm{y}$, de ser el embarazo posible, requiere un estricto control mediante ecografía.

Este síndrome se describió en todos los grupos étnicos; sin embargo, es conocido que la población amish de Lancaster (Pensilvania, Estados Unidos) es la más afectada. También se informaron varios casos en la India..$^{6-8}$ Cabe destacar que la madre y el padre del paciente provenían de una población de la selva peruana.

La condrodisplasia es la característica clínica más destacable y produce serios defectos en la osificación, por lo que las extremidades distales de los miembros son cortas y los pacientes son de baja estatura. ${ }^{1}$ En un estudio, la media de la desviación estándar en la talla para los varones y mujeres con SEVC, en comparación con la de los niños sanos, fue de $-3,1$ y -3 , respectivamente..$^{14} \mathrm{En}$ los adultos el rango de altura varía entre $132 \mathrm{~cm}$ y $155 \mathrm{~cm}$ en las mujeres, y entre $148 \mathrm{~cm}$ y $165 \mathrm{~cm}$ en los varones. ${ }^{12}$

El paciente del caso informado presentaba un florido cuadro de condrodisplasia; además, se incluía en el $50 \%$ al $60 \%$ de los casos que presentan malformaciones cardíacas.

La ecocardiografía mostró el compromiso descrito para el SEVC: aurícula única, defecto en la válvula tricúspide y comunicación interventricular. La anomalía cardíaca más frecuente es la aurícula única, observada en el $40 \%$ de los pacientes..$^{13}$ En el caso analizado, se sospecharon alteraciones cardíacas después que la radiografía de tórax sugiriera cardiomegalia. El compromiso cardíaco determina la supervivencia de los enfermos con SEVC. ${ }^{1}$

El paciente fue hospitalizado por un cuadro de dificultad respiratoria catalogado como neumonía, un factor vinculado al pronóstico de estos pacientes. La dificultad respiratoria en los primeros meses de vida está asociada a la estrechez torácica o a posibles defectos cardíacos. ${ }^{1}$ El tratamiento quirúrgico de las malformaciones congénitas cardíacas puede ser exitoso, pero la mortalidad es alta (aproximadamente $45 \%$ ) y existe elevada probabilidad de morbilidad respiratoria en el posoperatorio. ${ }^{15}$

Figura 2. A. Radiografía de tórax: aumento del tamaño de la silueta cardíaca. B. Ecocardiograma bidimensional; proyección apical de cuatro cámaras. Obsérvese la ausencia completa de septo interauricular que condiciona la presencia de una aurícula única (au); comunicación interventricular (civ). C. Ecocardiograma bidimensional; se observa la atresia tricuspídea (at. tric.).

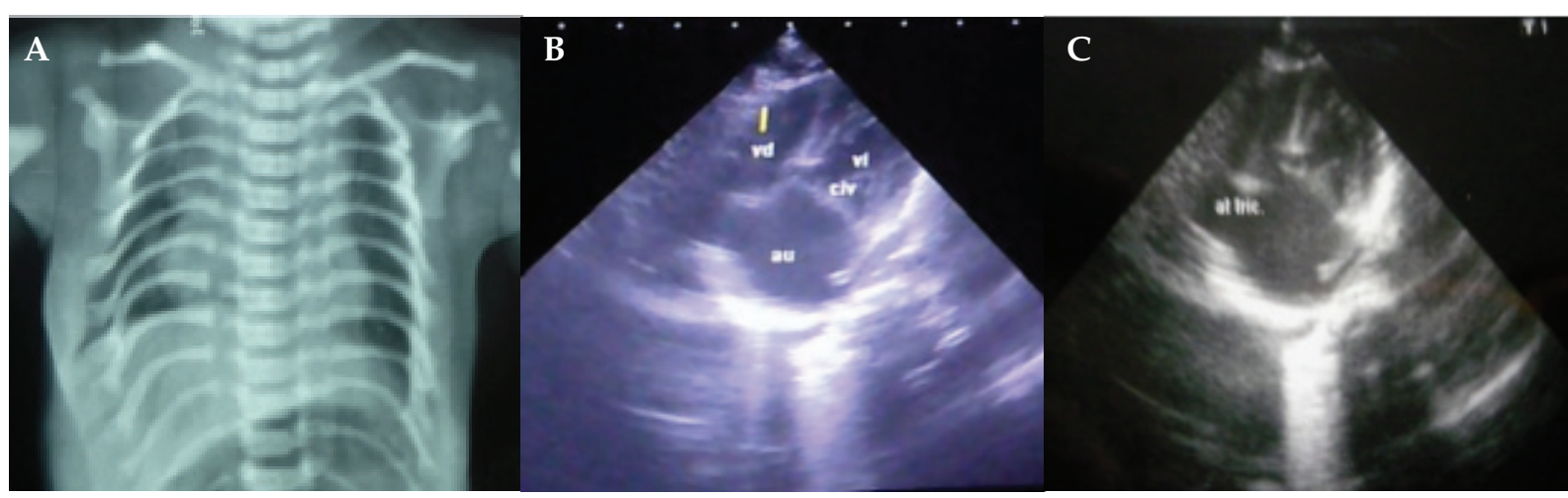


Las manifestaciones bucales son múltiples y se describieron ampliamente en los informes de casos. ${ }^{1,27}$ En este paciente se destacan las adherencias labiogingivales y la fusión del labio superior con el paladar, además de la pérdida prematura de dientes prenatales. Es necesario realizar más evaluaciones a fin de determinar el compromiso de la cavidad bucal y sus anexos.

El diagnóstico diferencial comprende los síndromes de costillas cortas-polidactilia, a menudo letales y con características propias: síndrome de Saldino-Noonan (tipo I), síndrome de Majewski (tipo II), síndrome de Verma-Naumoff (tipo III), síndrome de Beemer-Langer (tipo IV) y distrofia de Jeune, y otras causas más frecuentes de enanismo, como la acondroplasia y los síndromes bucofaciales. En el caso analizado, la presencia de malformaciones cardíacas apoyó el diagnóstico clínico de síndrome de Ellis-Van Creveld. 1,7,10,11

Según nuestra búsqueda en la bibliografía, este sería el primer caso peruano documentado y difundido en la comunidad científica como informe de caso. Su valor educativo radica en que presenta la tétrada clínica clásica del síndrome. A pesar de las limitaciones para confirmar el diagnóstico mediante el estudio genético, el cuadro clínico es compatible con lo descrito en la literatura especializada (la gran mayoría de los informes y series de casos publicados corresponden al diagnóstico clínico e imagenológico). ${ }^{6-9}$

\section{BIBLIOGRAFÍA}

1. Baujat G, Le Merrer M. Ellis-Van Creveld síndrome. Orphanet J Rare Dis 2007;2:27.

2. Alves-Pereira D, Berini-Aytés L, Gay-Escoda C. Ellis-Van
Creveld Syndrome. Case report and literature review. Med Oral Patol Oral Cir Bucal 2009;14(7):E340-3.

3. Lichiardopol C, Militaru C. Ellis-van Creveld syndrome. Rom J Morphol Embryol 2006;47(4):363-6.

4. Graziadio C, Bernardi P, Rosa RFM, Zen PRG, Paskulin GA. Type 1 diabetes in a patient with Ellis-van Creveld syndrome. Sao Paulo Med J 2012;130(1):53-6.

5. Estrada TT, Montoya VJH, Cortés-Yepes H. Prenatal diagnosis of Ellis-van Creveld syndrome: case report and literature review. RCOG 2011;62(3):250-4.

6. Veena K, Jagadishchandra $\mathrm{H}^{*}$, Kumar P, Chatra L. Ellisvan Creveld syndrome in an Indian child: a case report. Imaging Sci Dent 2011;41:167-70.

7. Vinay C, Reddy S, Ullopi K, Sekhar R. Clinical manifestations of Ellis-Van Creveld syndrome. J Indian Soc Pedod Prevent Dent 2009;4(27):256-9.

8. Kurian K, Shanmugam S, Harsh Vardah T, Gupta S. Chondroectodermal dysplasia (Ellis-Van Creveld syndrome): a report of three cases with review of literature. Indian J Dent Res 2007;18(1):31-4.

9. Mehndiratta S, Tyagi A, Devgan V. Ellis-van Creveld syndrome: report of two cases. World J Pediatr 2011;7(4):368-70.

10. Hills C, Kochilas L, Schimmenti L, Moller J. Ellis-van Creveld syndrome and congenital heart defects: presentation of an additional 32 cases. Pediatr Cardiol 2011;32(7):977-82 .

11. O'Connor MJ, Rider NL, Thomas Collins R, Hanna BD, Holmes Morton D, Strauss KA. Contemporary management of congenital malformations of the heart in infants with Ellis-van Creveld syndrome: a report of nine cases. Cardiol Young 2011;21(2):145-52.

12. Rudnik-Schöneborn S, Zerres K, Graul-Neumann L, Wiegand S, Mellerowicz H, Hehr U. Two adult patients with Ellis-van Creveld syndrome extending the clinical spectrum. Mol Syndromol 2010;1:301-6.

13. Shilpy S, Nikhil M, Samir D. Ellis Van Creveld syndrome. J Indian Soc Pedod Prevent Dent 2007;(Supp):S5-S7.

14. Verbeek S, Eilers P, Lawrence K, Hennekam R, Versteegh F. Growth charts for children with Ellis-van Creveld syndrome. Eur J Pediatr 2011;170:207-11.

15. Connor M, Rider N, Collins T, Hanna B, Holmes D, Strauss K. Contemporary management of congenital malformations of the heart in infants with Ellis-van Creveld syndrome: a report of nine cases. Cardiol Young 2011;21(2):145-52. 